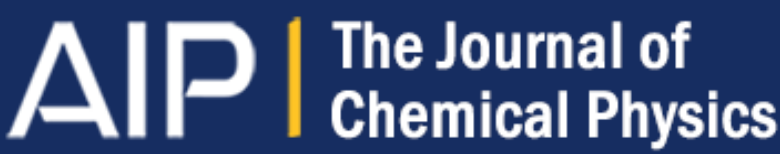

Reassessment of structure of smectic phases: Nano-segregation in smectic E phase in 4-n-alkyl-4-isothiocyanato-1,1-biphenyls

Kazuya Saito, Takahito Miyazawa, Akio Fujiwara, Mafumi Hishida, Hideki Saitoh, Maria Massalska-Arod, and Yasuhisa Yamamura

Citation: The Journal of Chemical Physics 139, 114902 (2013); doi: 10.1063/1.4821162

View online: http://dx.doi.org/10.1063/1.4821162

View Table of Contents: http://scitation.aip.org/content/aip/journal/jcp/139/11?ver=pdfcov

Published by the AIP Publishing

\section{AlP Re-register for Table of Content Alerts}




\title{
Reassessment of structure of smectic phases: Nano-segregation in smectic E phase in 4- $n$-alkyl-4'-isothiocyanato-1,1'-biphenyls
}

\author{
Kazuya Saito, ${ }^{1, a)}$ Takahito Miyazawa, ${ }^{1}$ Akio Fujiwara, ${ }^{1}$ Mafumi Hishida,${ }^{1}$ Hideki Saitoh, ${ }^{2}$ \\ Maria Massalska-Arodź, ${ }^{3}$ and Yasuhisa Yamamura ${ }^{1}$ \\ ${ }^{1}$ Department of Chemistry, Faculty of Pure and Applied Sciences, University of Tsukuba, Tsukuba, \\ Ibaraki 305-8571, Japan \\ ${ }^{2}$ Graduate School of Science and Engineering, Saitama University, Saitama 338-8570, Japan \\ ${ }^{3}$ The Henryk Niewodniczanski Institute of Nuclear Physics, Polish Academy of Sciences, \\ Kraków 31-342, Poland
}

(Received 13 July 2013; accepted 29 August 2013; published online 17 September 2013)

\begin{abstract}
Based on new diffraction data from aligned samples of smectic E (SmE) phase of 4- $n$-alkyl-4'isothiocyanato-1,1'-biphenyls, systematics against the alkyl chain length $n$ is analyzed. In order to perform the analysis, the molecular form factor approximated by a box-shaped distribution is calculated while taking the rounding of the distribution at corners into account. The analysis clearly shows the nano-segregated layered structure, which does not fit to the traditional structural view of SmE phase but does fit to the model the present authors proposed recently. Some implications of this conclusion are discussed in relation to the importance of the molten state of alkyl chains in most of real mesogens revealed previously through thermodynamic analyses. (C) 2013 AIP Publishing LLC. [http://dx.doi.org/10.1063/1.4821162]
\end{abstract}

\section{INTRODUCTION}

Smectic E (SmE) phase is a mesophase located in between the isotropic liquid and fully ordered crystal of rodlike (calamitic) molecules. The SmE phase is also known as crystal $\mathrm{E}(\mathrm{CrE})$ phase because the spatial periodicity survives in three dimensions with definite lattice constants within the orthorhombic system. ${ }^{1}$ Molecular orientation is highly disordered, which means the loss of distinction between the head and tail of the rodlike molecule due to the so-called flip-flop motions. Small anisotropy is, however, retained around the molecular long axis. In average, a molecule can be modeled by a rectangular parallelepiped with the point group symmetry of $D_{2 h}$. It has been widely accepted that molecules form the so-called herringbone array with the parallel alignment to one edge of the unit cell of crystal lattice, which is usually taken parallel to the $c$-axis. The widely accepted structural model is shown in Fig. 1. The model can be regarded as an anisotropic analog of "plastic crystal," 2 which usually has cubic or rhombic symmetry. The residual entropy determined calorimetrically ${ }^{3}$ is compatible with the model. It is also widely accepted ${ }^{4,5}$ that the excellent electrical property (high hole/electron mobility) of SmE compounds reported previously ${ }^{6}$ are based on this high degree of structural order.

SmE phase is the liquid-crystalline (LC) mesophase closest to (fully) ordered crystals. The present authors have previously revealed that alkyl chain(s) attached to mesogenic core are molten in any liquid crystalline phases, including the SmE phase. ${ }^{7-10}$ The molten chains stabilize LC phases through a large contribution in entropy term in Gibbs energy, and brings nano-segregated LC phases [such as SmA phase in $n \mathrm{CB}^{11}$ and

\footnotetext{
a)Electronic mail: kazuya@chem.tsukuba.ac.jp
}

cubic phases in $\operatorname{ANBC}(n)$ and $\left.\operatorname{BABH}(n)^{12}\right]$ by serving as an intramolecular solvent (self-solvent).

The present authors have pursued the last view while naming it the quasi-binary (QB) picture of thermotropics. ${ }^{13,14}$ During our experimental assessment on QB picture, we became suspicious on the widely accepted molecular arrangement in SmE phase shown in Fig. 1, because a binary system between $n$-nonane and 4- $n$-alkyl-4'-isothiocyanato-1, $1^{\prime}$ biphenyl (abbreviated as $n$ TCB with $n$ being the number of carbon atoms in the alkyl chain, shown in Fig. 2 for $n=4$ ), on which the largest number of studies has been reported as a representative of SmE mesogens, ${ }^{3,7,8,15-28}$ exhibits a swollen SmE phase in a continuous range of composition. ${ }^{29}$ Only the $c$, the cell constant parallel to the molecular long axis in average, exhibits a notable increase in swelling while retaining the three-dimensional periodicity. If the traditional model of molecular packing (Category II according to Diele et al ${ }^{30}$ ) is accepted, two possibilities are raised. One is that molecules of 9TCB and $n$-nonane, which are quite different in their shape and flexibility, freely mix to each other while keeping the lattice periodicity. The other is that $n$-nonane forms liquid layers between 9TCB layers. Both structures are hard to explain the experimental findings. ${ }^{29}$ Besides, the uniformly mixed layer of aromatic cores and chains is unfavorable for the electronic/hole conduction within the commonsense of organic conductors $^{31}$ that $\pi$-electrons (holes) on aromatic parts are responsible for the conduction and that the electronic interaction between aromatic parts, i.e., the overlap of wave functions of $\pi$-electrons ( $\pi$-orbitals) of neighboring molecules, is essential. The situation becomes more serious if the length of the alkyl chain is longer than that of the core part of a mesogen having a core-chain structure. Putting the cores at both ends of neighboring molecules in Fig. 1, no overlap of $\pi$-orbitals is realized. It contradicts the excellent mobility 


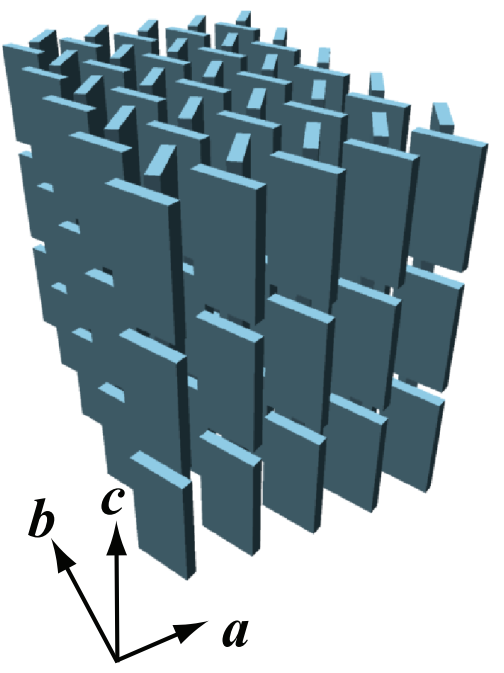

FIG. 1. Schematic illustration of widely accepted model of SmE phase. Molecules have $D_{2 h}$ symmetry, implying distinction between neither its head and tail nor the front and rear.

exhibited by SmE phase. ${ }^{6}$ Considering these difficulties, in a previous paper, ${ }^{29}$ the present authors proposed a new model involving nano-segregation between aromatic cores and alkyl chains.

A serious attempt to reveal a structure of SmE phase, the layer spacing of which is apparently longer than the molecular length, was reported previously ${ }^{32}$ without paying attention to systematics. On the other hand, the present authors have shown that the systematics is crucial for studies on complex systems such as LCs. ${ }^{12,33-38}$ Indeed, the phase problem for a bicontinuous Gyroid phase was solved while taking into account the systematic variation of reflection intensities with the alkyl chain length. ${ }^{12,37}$ In this paper, we attempt to establish the validity of the model proposed in a previous paper $^{29}$ for SmE phase through the analysis of systematics against chain-length $n$ in the X-ray diffraction intensity of neat $n \mathrm{TCB}$. Implications of the aggregation struc-

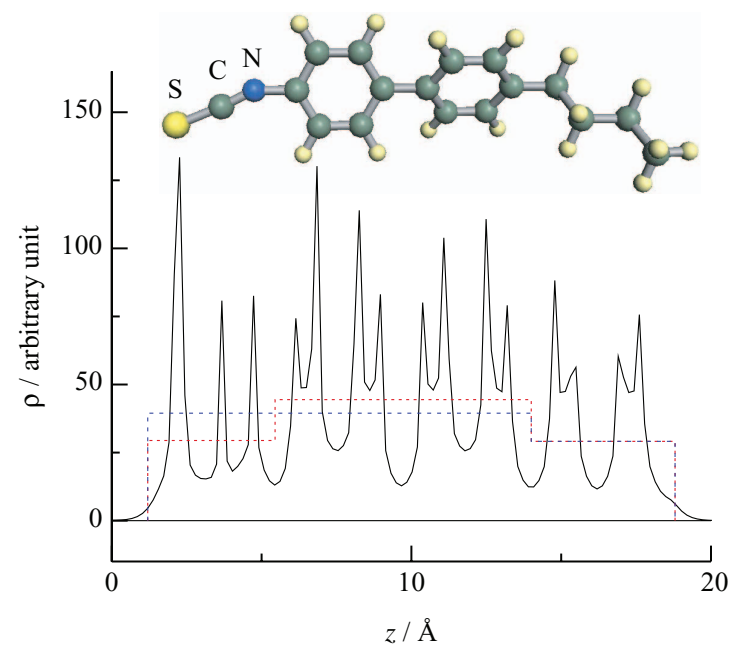

FIG. 2. Molecule of 4TCB and electron density projected on $z$-axis obtained through a quantum-chemical optimization in energy. Dotted and dashed lines represent the average box-shaped density assuming three and two regions, respectively. ture are discussed in relation to the intrinsic lyotropic nature of thermotropic smectics. ${ }^{11,39}$ We concentrate ourselves to the structure along the $c$-axis. The structure inside the $a b$ layer is beyond the scope of the present paper. Note, however, that the model has a mechanism that naturally explains threedimensional periodicity of the SmE phase. ${ }^{29}$

This paper is organized as follows: Sec. II deals with the molecular form factor of a $n$ TCB molecule through quantum chemical calculation. In Sec. III, the experimental results are presented. Section IV demonstrates the nano-segregation through the analysis of systematics and Fourier syntheses. Concluding remarks are given in Sec. V. The Appendix describes the crystal structure of 9TCB. Details of calculation of and discussion on the form factor for box-shaped electron density are given in the supplementary material. ${ }^{40}$

\section{MOLECULAR FORM FACTOR OF $n$ TCB}

To get insights on electron density distribution over an $n$ TCB molecule, a quantum-chemical calculation was performed on 4TCB at the B3LYP/6-31G level using Gaussian $09 .{ }^{41}$ The result is shown in Fig. 2. In accordance with the widely accepted view, the electron density on the central aromatic region (biphenyl) is higher than that on the alkyl chain.

Since the orientation and conformation (and even the position as revealed later) of molecules are highly disordered in SmE phase, atomic details should be averaged into some coarse-grained profile of electron density. Due to a high density on the sulfur atom, it is not so obvious how we should treat that on the NCS group in comparison with those on the aromatic and chain regions. The physical/chemical commonsense is to be taken into account at this point. Considering the significant difference in shape and flexibility, we assume two types of differentiation: the division of electron distribution into three parts (chain, biphenyl core, and NCS group) and into two parts (chain and "core + NCS"). Either of the division methods is to be adopted on the basis of the better plausibility upon comparing the experimental and calculated diffraction intensities. The results of two methods of averaging the electron density over each part are shown by broken or dashed lines in Fig. 2. The division into three parts gives quite a similar average density for the NCS group and the alkyl chain. This means that the electron density is essentially approximated by a two-valued, two-step function. The division into two parts, needless to say, gives a two-valued, single-step density.

Although $c$ has been reported to be slightly longer than the molecular length, the sum of van der Waals radii of the outermost atoms and the distance in between obtained quantum-chemically well coincide with it. Besides, the small gap in electron density between molecular layers should give strong X-ray diffraction at high angles [constant intensity in the case of zero-width gap ( $\delta$-function) in diffractions of any order], in strong contrast to experimental diffraction profiles of SmE phases as will be shown later. Consequently, the gap in electron density between molecular layers is assumed to be absent. Then, the two-valued, two-step function in the division into two parts can be rearranged into a single-step function. Hence, both ways of the division of a molecule upon 
averaging yield a single-step function. The difference is in relative length of high and low density parts. In fact, the deviation into two parts seems to approximate better the reality as described later on the basis of the experimental systematics.

\section{EXPERIMENT AND RESULTS}

All samples of $n$ TCB used in the present study were supplied by Professor R. Dabrowski, the Military University of Technology in Warsaw, Poland, and used as obtained. Previous calorimetric studies ${ }^{3,7}$ on some of the samples suggest that their purities are 99 mol.\% at the worst. Wide-angle Xray diffraction experiments were performed using a Rigaku Ultima IV powder diffractometer $(\mathrm{CuK} \alpha, \lambda=1.5419 \AA)$. The temperature of the sample was controlled using a hightemperature stage equipped with a cartridge heater attached to a laboratory-made sample-plate holder, which is well isolated from the goniometer. The temperature was monitored by a thermocouple (type E), the junction of which was placed near the sample.

Since the present study focusses on the stacking structure of layers in SmE phase, samples were homeotropically aligned by melting them once in air on the sample holder set on the diffractometer. By this method, reflections up to the fifth order (in the best case) were observed as shown in Fig. 3. Intensity of each reflection was evaluated with respect to $I(001)$ as exemplified in Table I. Relative intensities to $I(001)$ of each sample were confirmed to be stable against temperature variation except for temperatures very close to the transition temperature to the isotropic liquid. Their systematics as functions of the layer spacing is shown in Fig. 4. To avoid unexpected influence of temperature, in the present paper the systematics is discussed at $333 \mathrm{~K}$, which is rather high and lower than the transition temperature by ca. 3-5 K.

For meaningful comparison between experimental and calculated intensities, some correcting factors should be taken into account such as a Lorentz-polarization factor $\left(L_{\mathrm{p}}(\theta)\right)$ and/or correction for the dispersion of X-ray beam. Fortunately, the ratio for different $l$ remains constant within a few percent against the chain-length $n$ by virtue of a small scattering angle $\theta$ for $\{00 l\}$ because of $\theta(l) \approx l \cdot \theta(1)$, and $L_{\mathrm{p}}(\theta) / L_{\mathrm{p}}(2 \theta) \approx 2$ for powder samples, for example. Hence, the chain-length dependence can be compared without diffi-

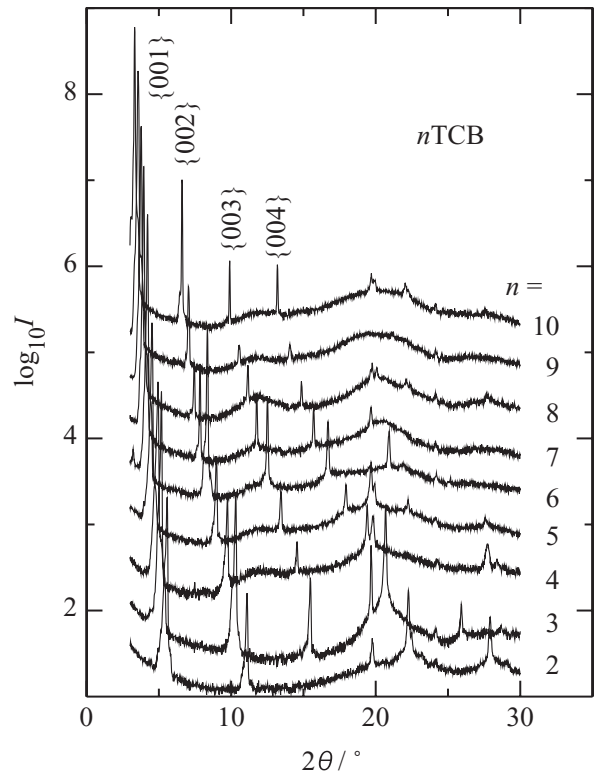

FIG. 3. Experimental X-ray diffraction patterns from aligned $n \mathrm{TCB}$ at 333 K. Data of $n=3-10$ are successively shifted by $0.5\left(=\log _{10} 10^{\frac{1}{2}}\right)$ upward.

culty between the experimental and calculated diffraction intensities if plotted in a logarithmic scale.

\section{STRUCTURE OF SMECTIC E PHASE}

\section{A. Structure factor of SmE phase and molecular electron density distribution}

Considering that the molecular electron density $\rho(z)$ is essentially of the box-shape (with finite rounding at corners), we assume

$$
\rho(z)=\frac{\rho_{0}}{2}\left\{h\left[\frac{2}{r}\left(z+\frac{w}{2}\right)\right]-h\left[\frac{2}{r}\left(z-\frac{w}{2}\right)\right]\right\}
$$

with

$$
h(z)=\tanh z
$$

The width of the box and the length characterizing the rounding are $w$ and $r$, respectively. As detailed in the supplementary material, ${ }^{40}$ the resultant molecular form factor at a diffraction

\begin{tabular}{|c|c|c|c|c|c|c|}
\hline & $c(\AA)$ & $I(002) / I(001)$ & $I(003) / I(001)$ & $I(004) / I(001)$ & $I(005) / I(001)$ & $\operatorname{Sign}^{\mathrm{a}}$ \\
\hline $2 \mathrm{TCB}$ & 15.974 & $7.6 \times 10^{-2}$ & & $8.5 \times 10^{-2}$ & $4.3 \times 10^{-2}$ & +-+-- \\
\hline 3ТСB & 17.185 & $8.2 \times 10^{-2}$ & $8.4 \times 10^{-3}$ & $7.7 \times 10^{-2}$ & $4.3 \times 10^{-3}$ & +-++- \\
\hline $4 \mathrm{TCB}$ & 18.237 & $4.0 \times 10^{-2}$ & $8.8 \times 10^{-3}$ & $2.6 \times 10^{-2}$ & $\ldots$ & +-++- \\
\hline $5 \mathrm{TCB}$ & 19.756 & $3.3 \times 10^{-2}$ & $8.2 \times 10^{-3}$ & $1.1 \times 10^{-2}$ & $1.1 \times 10^{-3}$ & +--+- \\
\hline $6 \mathrm{TCB}$ & 21.233 & $5.5 \times 10^{-2}$ & $1.0 \times 10^{-2}$ & $6.1 \times 10^{-3}$ & $3.3 \times 10^{-3}$ & +--++ \\
\hline 7ТCB & 22.553 & $5.7 \times 10^{-3}$ & $2.6 \times 10^{-3}$ & $1.8 \times 10^{-3}$ & $1.3 \times 10^{-3}$ & +--++ \\
\hline $8 \mathrm{TCB}$ & 23.827 & $2.0 \times 10^{-3}$ & $1.5 \times 10^{-3}$ & $7.1 \times 10^{-4}$ & $\ldots$ & +--++ \\
\hline 9ТСВ & 25.208 & $3.7 \times 10^{-3}$ & $4.4 \times 10^{-4}$ & $4.9 \times 10^{-4}$ & $\ldots$ & ++--+ \\
\hline 10TCB & 26.825 & $1.6 \times 10^{-2}$ & $1.6 \times 10^{-3}$ & $1.5 \times 10^{-3}$ & $\ldots$ & ++--+ \\
\hline
\end{tabular}

TABLE I. Experimental diffraction intensities normalized by $I(001)$ of $n \mathrm{TCB}$ at $333 \mathrm{~K}$.

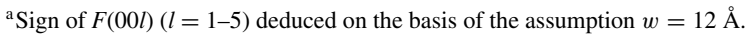




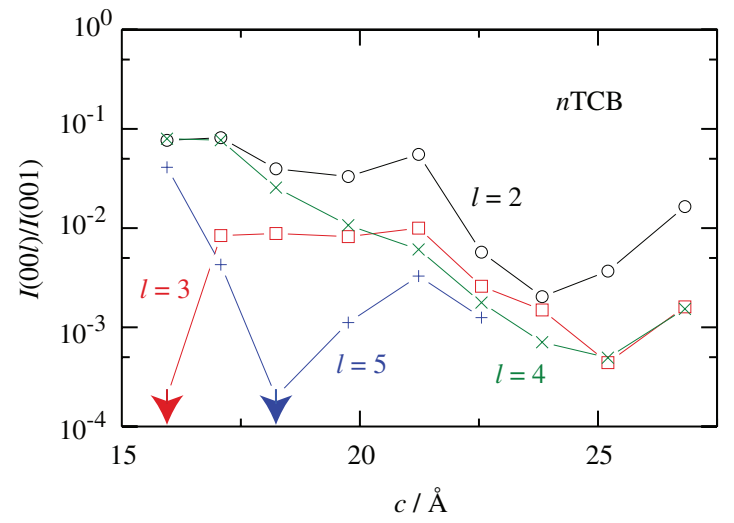

FIG. 4. Systematics of experimental $I(00 l) / I(001)$ of $n \mathrm{TCB}(n=2-10)$ at $333 \mathrm{~K}$. Circle, $l=2$; square, $l=3$; cross, $l=4$; and plus, $l=5$. Arrows indicate the intensity was below the detection limit.

vector $q$ has the following form:

$$
f(q, w, r)=\frac{2 \rho_{0}}{q} \sin \frac{q w}{2} \cdot R(q r),
$$

with a reduction factor $R(q r)$ due to the rounding,

$$
R(q r)=\frac{\pi q r}{4 \sinh \frac{\pi q r}{4}} .
$$

Here, we write the molecular form factor as $f(q, w, r)$ to indicate its dependence explicitly on $w$ and $r$. The above form factor has two properties relevant to the analyses in this paper. First, as evident from the above expression, it is factorized as the product of that of the ideal box (without rounding) and the reduction factor due to rounding. Second, $R(q r)$ does not change the sign. This means that the sign of $f(r, w, r)$ solely depends on the form factor of the ideal box. Note that the choice of the functional shape for the rounded step function ( $\tanh z$ in this case) is not essential for the following discussion (see the supplementary material ${ }^{40}$ ).

Since SmE phase has the inversion symmetry, the unit cell having two molecules inside can be modeled by the superposition of electron densities of two molecules with opposing orientations. Packing modes considered in this paper are schematically shown in Fig. 5. The average structure of the 1D SmE crystal is thus the superposition of electron densities arising from two molecules with a finite shift $d$ between them. Its structure factor $F(q)$ is given by

$$
F(q)=2 \cos \frac{q d}{2} \cdot f(q, w, r)
$$

while putting two boxes at $z= \pm d / 2$. The intensity of diffracted X-ray is given by the square of magnitude of this structure factor. It is emphasized that the structure factor given by Eq. (4) is factorized to the product of factors involving separately $w$ (width of the box), $r$ (rounding of corners), and $d$ (relative shift of two boxes within a unit cell).

According to the above expression of $F(q), F(00 l)$ is null if $f(q, w, r)$ vanishes. Equation (2) indicates that this occurs for $w / c=k / l$ with $k \in N$, irrespective of $d$. If $F(001)$ is nonvanishing, this offers the way to discriminate either of two divisions of a molecule into a step function, as follows. According to the quantum-chemical calculation, the length of i-a

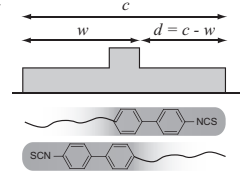

ii-a
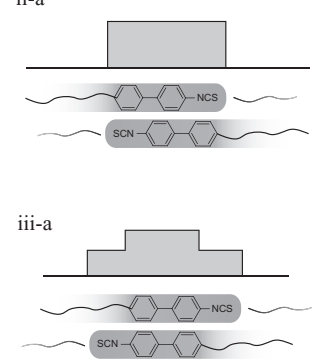

i-b

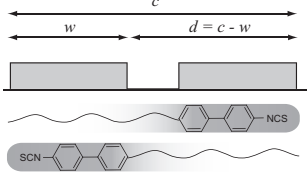

ii-b

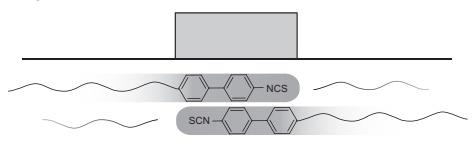

iii-b

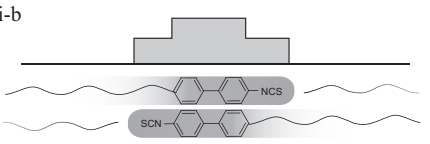

FIG. 5. Probable modes of molecular arrangement (in average) in SmE phase of $n \mathrm{TCB}$ with the layer spacing $c$ shorter (a) or longer (b) than the twice of the part with higher electron density (NCS + biphenyl). (i) Traditional model, (ii) alkyl chain separated from other parts, and (iii) nano-segregation due to the difference in flexibility.

aromatic core is ca. $8.6 \AA$. Since $F(002)$ is normally observed for 3TCB with $c=17.4 \AA$ as seen in Figs. 3 and 4, the division of a molecule into three parts shown in Fig. 2 is to be discarded. On the other hand, the division into two parts seems consistent with the experimental observation that $F(002)$ is very weak for $n=8(c=23.8 \AA)$ and $n=9(c=25.2 \AA)$ because the length of the high-density part (NCS + biphenyl) is ca. $12 \AA$. The division of molecular electron density into two parts is therefore adopted in the followings. Reflections with higher indexes in discretion between $w=12 \AA$ and $w=8.6 \AA$ is now considered for little, since they are more sensitive to the non-ideality (deviation from the ideal rectangular box).

Equation (4) also shows that $F(00 l)$ vanishes if $d / c$ $=(2 k+1) / 2 l$ with an arbitrary integer $k$ irrespective of the detail of the molecular form factor $f(q, w, d)$. Since $0 \leq d$ $\leq c / 2$ can be assumed without the loss of generality, the condition has meaning for $0<2 k+1<l$. It is interesting to note the experimental fact that $I(003)$ is missing for $n=2(c=16.0 \AA)$. If this absence arises from this mechanism, $d$ is $2.67 \AA$ or $8.0 \AA$.

In Subsections IV B and IV C, we compare the experimental reflection intensities $I(00 l)$ with theoretical ones. Based on the above discussion, we adopt Eq. (1) as the molecular electron density distribution with $w=12 \AA$ and $r=3 \AA$. The shift $(d)$ of two molecules in average in a unit cell varies depending on the packing model assumed. In the comparison, $I(00 l) / I(001)=|F(00 l) / F(001)|^{2}$ are mainly used because the measurement of absolute intensity is hard.

\section{B. Packing models}

To proceed further by comparing the model with experimental results, three cases seem to deserve consideration: (i) molecules form a perfect and separate layer in accordance with the commonly accepted structural model of SmE phase, (ii) alkyl chain and other part is nano-segregated due to the 


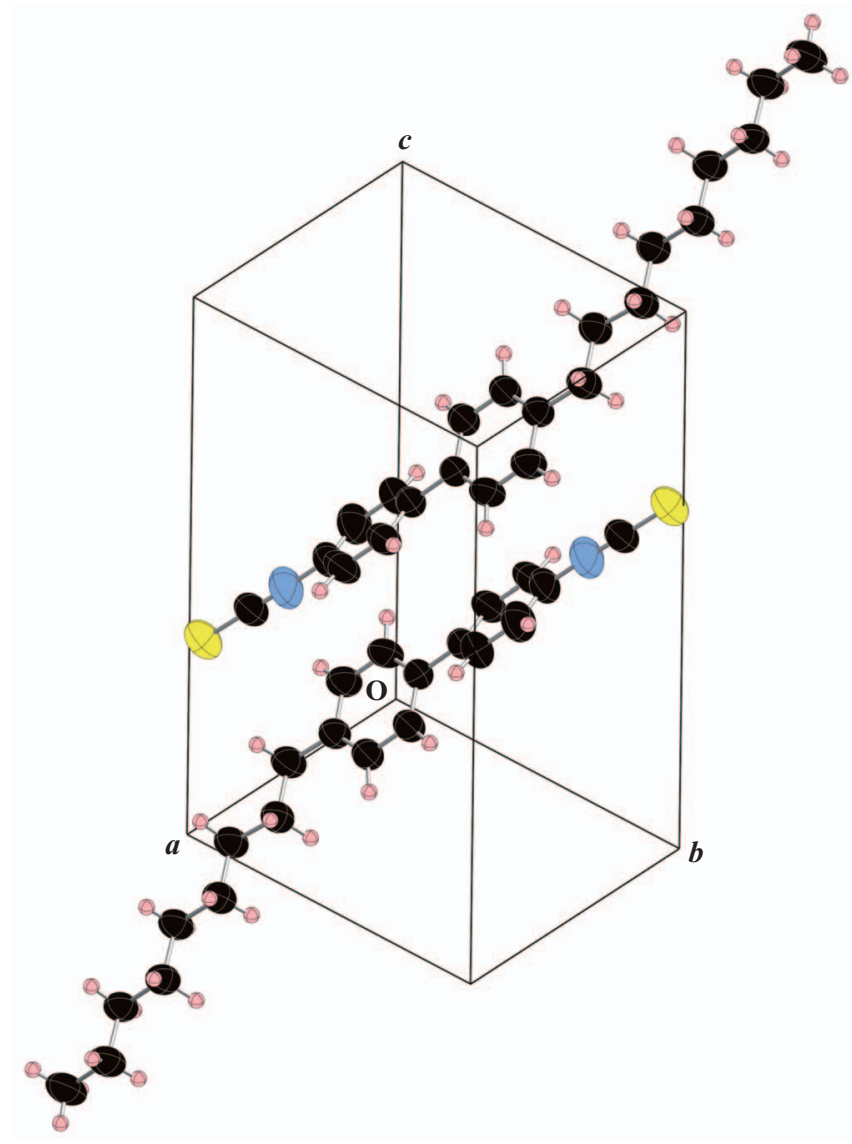

FIG. 6. Crystal structure of 9TCB at room temperature. Thermal ellipsoids of non-hydrogen atoms indicate the region of $50 \%$ probability while those of hydrogen atoms are not shown for clarity.

so-called chemical amphiphilicity, and (iii) nano-segregation occurs due to the difference in dynamics. Cases (ii) and (iii) correspond to a variation within the model proposed by the present authors in the previous paper. ${ }^{29}$ Case (ii) can also be expressed as the high electron density part (NCS + biphenyl) being laterally adjacent. Case (iii) would be stabilized by the chemical interaction (the so-called $\pi-\pi$ interaction between aromatic cores) because non-molten part of the alkyl group in the SmE phase has a length of ca. $3 \AA$ as deduced from thermodynamic analysis in previous papers. ${ }^{7-10}$ This structure is similar to that observed in the ordered crystal in 9TCB as shown in Fig. 6. Electron density distribution for each case is illustrated in Fig. 5 ignoring the rounding of electron density at corners.

First, we begin with case (ii) because it is exactly the same as the behaviors of box-shaped molecular form factor. In this case, the electron density distribution in a unit period is a two-valued and single-step function. To use Eq. (4), the origin $(z=0)$ should be placed at the center of the box. The width of the high electron density part remains the same as $w$ irrespective of the change in $c$. The dependence of the normalized reflection intensities on $c$ is shown in Fig. 7 [case (ii)].

In case (i), the electron density distribution in a unit period exhibits two types. For $c<2 w$, it is a two-valued function with a varied width of the high-electron density part $(2 w-c)$ as seen in Fig. 5(i-a). The origin $(z=0)$ should
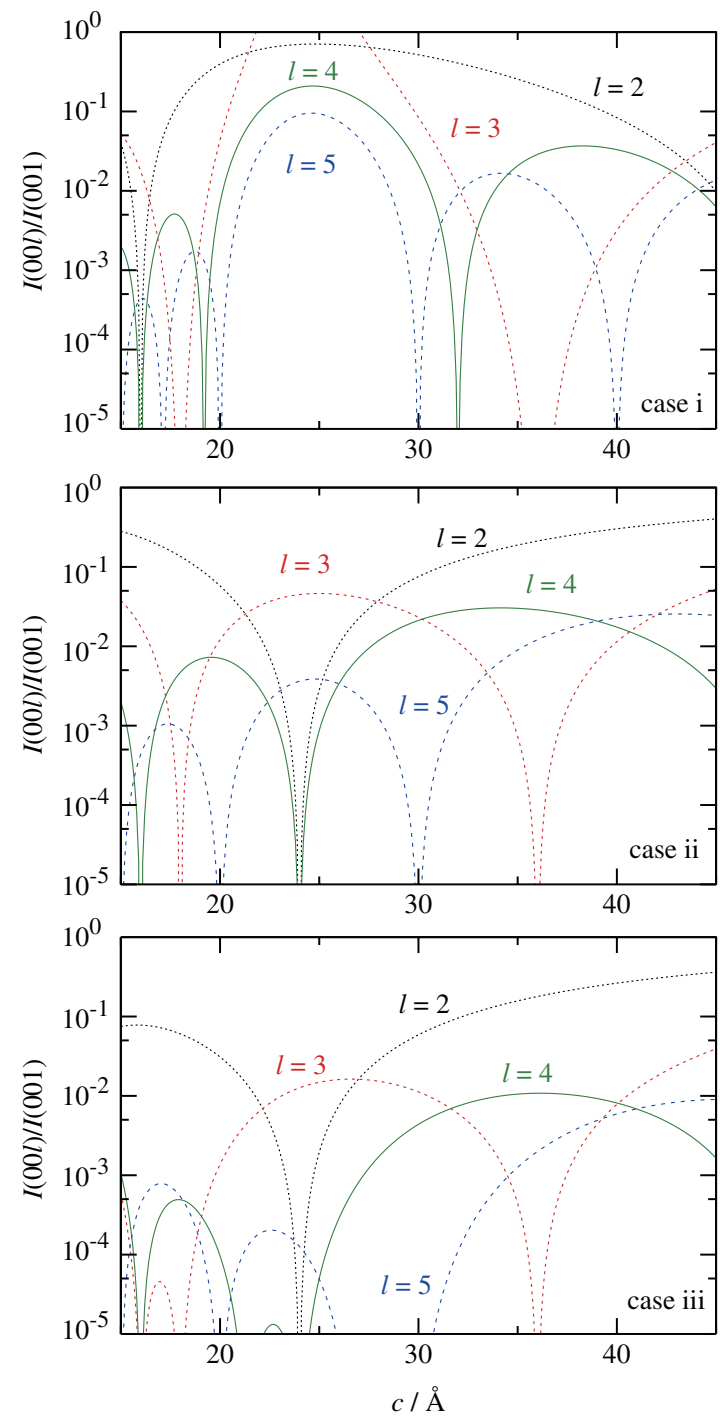

FIG. 7. Normalized theoretical intensities $I(00 l) / I(001)$ as a function of the layer spacing $c$ while assuming the molecular electron density being given by Eq. (1) with $w=12 \AA$ and $r=3 \AA$. Case i, $d=c-w$; case ii, $d=0 \AA$; and case iii, $d=2.67 \AA$.

be placed at the center of the combined electron density to adopt Eq. (4). On the other hand, for $c>2 w$ (case i-b), the electron density distribution is a two-valued function with the constant width of $2 w$ for the high electron density part with $d=w / 2$. In between these two types of electron density distributions, the uniform electron density is realized at $w / c=1 / 2$ irrespective of $r$. All $F(00 l)$ including $l=1$ vanish there as exemplified in Fig. 8 at $c=24 \AA$ ( $w=12 \AA)$, accordingly. Besides, reflecting this vanishing $F(001)$, the normalized intensities exhibit peculiar behaviors as shown in Fig. 7 [case (i)]. The intensities of the second and third order reflections are calculated as being of the same order as the fundamental $(l=1)$ around $c=24 \AA$.

Finally, the electron density distribution is always threevalued function in case (iii). The dependence of normalized theoretical intensities on $c$ is similar to those of case (ii). The distinction between cases (ii) and (iii) may depend on the relative shift $d$ and the degree of rounding of the electron density "box." 


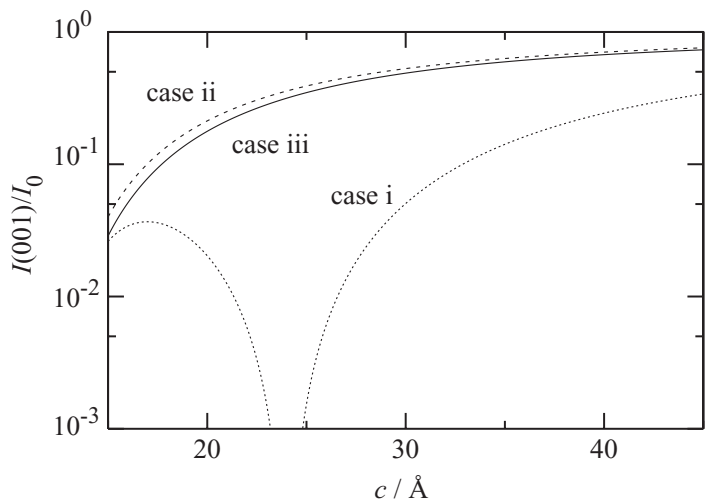

FIG. 8. Dependence of $I(001)$ (normalized by the possible maximum intensity $I_{0}=4 w^{2}$ ) on the layer spacing $c$ while assuming the molecular electron density being given by Eq. (1) with $w=12 \AA$ and $r=3 \AA$. Case i, $d=c-w$; case ii, $d=0 \AA$; and case iii, $d=2.67 \AA$.

\section{Systematics}

Although the quantitative statement is difficult, no anomalous dependence of $I(001)$ on the chain length such as a tendency to vanishing was experimentally recognized. This finding implies case (i) is not a good description, being irrespective of the assumed width of high electron density part of a molecule ( $8.6 \AA$ or $12 \AA$ ). Rather, a tendency of $F(002)$ for vanishing around $c=24 \AA$ suggests the width of the high electron density part of a molecule to be $12 \AA$. Also $F(00 l)$ with $n=2-5$ depends on $c$ differently from case (i). This clearly demonstrates that case (i) is inconsistent with the experimental results. On the other hand, except for clear indication of rather strong $I(004) / I(001)$ around $c=16 \AA(=4 w / 3)$ in the experimental result, the dependences of $I(00 l) / I(001)$ resemble case (ii) and/or (iii) in Fig. 7.

Beside this chain-length dependence in neat systems, the results of swollen SmE phase found in binary systems between $n \mathrm{TCB}$ and $n$-alkane are also of interest. In the binary systems, on heating, the amount of $n$-alkane in the swollen phase decreases, resulting in reduction in the layer spacing without the change in the periodicity within layers. Since $c$ can be changed without changing sample in contrast to the neat system, the absolute reflection intensities are discussed without normalization by $I(001)$. If the aggregation mode is of case (i), Fig. 8 shows that $I(001)$ increases significantly (up to 1 order of magnitude) upon swelling of SmE phase of 9TCB $(c=25.2 \AA)$. However, this was not observed. ${ }^{29}$

In summary, the systematics in neat series of $n \mathrm{TCB}$ and the dependence on the degree of swelling in 9TCB- $n$-nonane system are in contradiction to the traditional model of $\mathrm{SmE}$ phase [case (i)], and rather favor the nano-segregated aggregation in cases (ii) and (iii). Distinction between two cases is difficult as expected.

\section{Fourier synthesis}

Since the $I(002) / I(001)$ exhibits the tendency for vanishing at the reasonable value of the periodicity $c$, it seems reasonable to assume the distribution of the molecular electron density is approximated, even roughly, by a box with finite

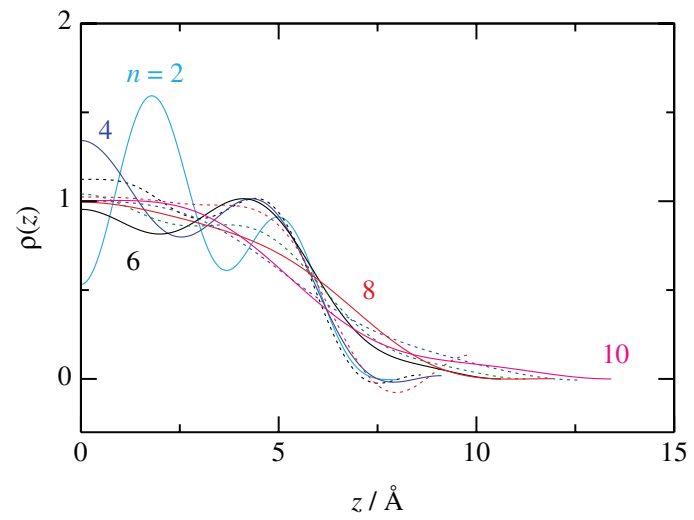

FIG. 9. Electron density obtained through Fourier synthesis. Curves are drawn in the range $0 \leq z \leq c / 2$. Solid curve, even $n$; dotted curve, odd $n$.

rounding at corners. As noticed previously, in this case, the relative phase of each reflection can be deduced on the basis of the sign of the molecular form factor (Eq. (2)). The deduced phases assuming $w=12 \AA$ are given in Table I.

Using the sign for each reflection thus deduced, electron density was naively obtained through Fourier syntheses

$$
\rho(z)=\sum_{l=1,5} \cos \left(\frac{2 \pi l}{c} z\right) \times[\operatorname{sign}] \sqrt{I(00 l)} \cdot L_{\mathrm{p}} .
$$

$L_{\mathrm{p}}=1 / \sin \theta$ was simply assumed. Essentially the same results were obtained through the maximum entropy analysis, which worked effectively to reveal molecular aggregation/arrangement in nano-segregated cubic LCs. ${ }^{12,42}$

Calculated electron densities are shown in Fig. 9. Since two plateaus of the electron densities were recognized, the lower plateau was, though roughly, set to zero while the scale was determined to give the constant integrated magnitude for every samples. Namely,

$$
\int_{0}^{c / 2} \rho(z) d z=\text { const. }
$$

The shown electron densities are thus scaled. The all electron densities drastically decrease at around $6 \AA$, which is exactly the half of the assumed core width $w=12 \AA$. The results indicate that the width of the high electron density part (core part) is almost constant and the low density part (chain part) is elongated with increasing $c$, i.e., the core and chain parts are nano-segregated. Note that the assumption in deducing the phase of each reflection is compatible with all cases considered above (i-iii). The resultant electron densities should suffice the distinction between case (i) and others, accordingly. Since neither systematic decrease in the width of the high value plateau on increasing $c$ for $c<2 w$, nor constant width of $2 w$ for $c>2 w$ (because the origin is displaced at $c=2 w$ ) is recognized, case (i) is again ruled out. Unfortunately, on the other hand, the distinction between cases (ii) and (iii) is still impossible. It is again emphasized that the structure of SmE phase of $n \mathrm{TCB}$ is described as the alternate stack of two types of layers: one with high electron density consisting mainly of biphenyl cores and NCS- groups, and one with lower density consisting of alkyl chains. This is just the structure assumed 
in the proposed model by the present authors in the previous paper. ${ }^{29}$

\section{CONCLUSION}

Based on diffraction data from aligned samples of SmE phase of neat $n \mathrm{TCB}$, the systematics against the chain length $n$ is analyzed. The analysis confirmed the model of SmE structure proposed by the present authors in a previous paper: ${ }^{29}$ alternately nano-segregated layered model, where the geometrical centers of molecules do not form layers. The structural model is compatible with the formation of the swollen SmE phase having the same periodicity within the layer. ${ }^{29}$ Also the structure is consistent with the excellent carrier mobility previously reported for $\mathrm{SmE}$ compounds, ${ }^{6}$ because the electron transfer is possible even in dynamically disordered state as long as moieties responsible for it are adjacent. ${ }^{43,44}$

The structure revealed in this study resembles the aggregation structure of $8 \mathrm{CB}$ in the SmA phase, ${ }^{45,46}$ in the segregation of chains and others. Although the segregation in $n \mathrm{CB}$ has long been interpreted as the result of strong interaction between electric dipole borne by the cyano (NC-) group attached to the core instead of SCN- group in $n \mathrm{TCB}$, the kinetic entropy arising from the difference in flexibility ${ }^{39}$ is to be considered as an important driving factor toward the nanosegregation, as in the depletion interaction ${ }^{47}$ and Alder transition of hard spheres. ${ }^{48}$ This is based on the fact that the alkyl chain of $n \mathrm{TCB}$ is already molten even in SmE phase to the same extent as in the isotropic liquid. ${ }^{7-10}$ Since SmE phase is the closest to ordered crystals among LC mesophases with orthogonal molecular order, the nano-segregation is considered to play important roles in any smectic phases exhibited by mesogens having long chain(s). It is noteworthy that the molecular aggregation in smectic phases would be largely different between seemingly symmetric (chain-core-chain) and asymmetric (chain-core) mesogens, accordingly, if described in terms of the molecular centers of gravity.

LCs have usually been treated, from the point of view of basic research, by dividing them into two groups: thermotropic and lyotropic. The former results from the shape anisotropy of constituting particle. ${ }^{4-51}$ The formation of smectic structure is understood to be driven by the kinetic entropy as two-dimensional liquid as shown by the results of molecular dynamics simulation on systems consisting of hard cylinders ${ }^{51}$ or spherocylinders. ${ }^{50}$ In contrast, the aggregation structure revealed in the present study does not allow two-dimensional fluidity because chains penetrate into neighboring layers. The loss in the kinetic entropy of molecular centers of gravity is compensated by the conformational entropy of the flexible chain. In this respect, it is interesting to note that the entropy of fusion of a crystal of rare gasses (Ne, $\mathrm{Ar}, \mathrm{Kr}$, and $\mathrm{Xe}$ ), which solely involves the entropy change in disordering of the positions of molecules (atoms in reality), has a common magnitude of $14 \mathrm{~J} \mathrm{~K}^{-1} \mathrm{~mol}^{-1}$, whereas the entropy "capacity" upon conformational melting of alkyl chain amounts to ca. $10 \mathrm{~J} \mathrm{~K}^{-1}$ (mol of $\left.\mathrm{CH}_{2}\right)^{-1}$. ${ }^{33}$ This magnitude does not fully contribute to the gain of the conformational entropy upon nano-segregation, but does as a mass. The degree of penetration of chains into neighboring layers would vary depending on mesogens, and possibly explain a variety observed for the anisotropy of molecular diffusion in smectic LCs. ${ }^{52}$

Finally, a comment is given concerning the significance of this work. The authors believe that this work shed light on the "liquid crystallinity" of SmE phase, which has often been debated because three-dimensional order certainly exists in some extent. Note that there is, as far as the authors' knowledge, no simple molecular model explaining the appearance of SmE phase, where a harder head-to-tail disorder is achieved whereas an easier one around a molecular long axis does not, resulting in the survival of the intralayer anisotropy. The present study demonstrates that the SmE phase is not an anisotropic analog of the plastic crystal ${ }^{2}$ but a LC aggregate having a lamellar structure. That is, it can be understood correctly and coherently only when its liquid crystallinity is adequately taken into account. The authors hope this conclusion contributes to the progress of understanding of various non-conventional LCs because SmE phase is the closest to ordinary crystals.

\section{ACKNOWLEDGMENTS}

The authors are deeply indebted to Dr. W. Press for his comments and suggestion during a memorial conference "Neutron Spectroscopy and Related Phenomena - Tribute to Jerzy Janik" held on March 21 and 22, 2013 at Kraków, Poland. This work was supported in part by Grant-in-Aid for Scientific Research (B) 22350056 from Japan Society for the Promotion of Science (JSPS). Experiment at Photon Factory, High Energy Accelerator Research Organization (KEK), Japan was performed under the Project No. 2012G636.

\section{APPENDIX: STRUCTURE OF ORDERED CRYSTAL OF 9TCB}

Single crystals of 9TCB were grown from toluene solution at room temperature. Single-crystal diffraction experiments were performed at BL-8A of Photon Factory, KEK, Japan using the synchrotron radiation monochromated by two silicon monochromators. The diffraction intensities were measured at room temperature using a cylindrically curved imaging plate (IP). Data reduction and cell refinement were performed by PROCESS-AUTO. ${ }^{53}$ The structure was solved by the direct method using SIR $88,{ }^{54}$ expanded by difference Fourier syntheses, and refined by SHELXL97. ${ }^{55}$ The

TABLE II. Crystal data of 9TCB at $301 \mathrm{~K}$.

\begin{tabular}{lll}
\hline \hline Empirical formula & $\mathrm{C}_{22} \mathrm{H}_{27} \mathrm{~N}_{1} \mathrm{~S}_{1}$ & \\
Formula weight & 337.52 & \\
Crystal system & Triclinic & \\
Space group & $P \overline{1}$ & \\
Unit cell & $a=6.9093(2) \AA$ & $\alpha=95.1161(9)^{\circ}$ \\
& $b=9.2123(2) \AA$ & $\beta=102.2560(9)^{\circ}$ \\
& $c=16.1838(7) \AA$ & $\gamma=101.7660(9)^{\circ}$ \\
& $V=975.99(6) \AA^{3}$ & \\
$Z$ & 2 & \\
Density (calculated) & $1.148 \mathrm{Mg} \mathrm{m}^{-3}$ & \\
\hline \hline
\end{tabular}


TABLE III. Summary of structure refinement for 9TCB at $301 \mathrm{~K}$.

\begin{tabular}{ll}
\hline \hline Absorption coefficient & $0.406 \mathrm{~mm}^{-1}$ \\
$F(000)$ & 364 \\
Radiation & Synchrotron \\
Wavelength & $0.9995 \AA$ \\
Crystal size & $(0.22 \times 0.14 \times 0.10) \mathrm{mm}^{3}$ \\
$\theta$ range & $3.66^{\circ}<\theta<45.70^{\circ}$ \\
Index range & $-9<h<9$ \\
& $-10<k<7$ \\
& $-23<l<16$ \\
Reflection collected & 4924 \\
Independent reflections & $3557\left(R_{\text {int }}=0.0155\right)$ \\
Completeness to $45.55^{\circ}$ & $59.8 \%$ \\
Absorption correction & Not applied \\
Refinement method & Full-matrix least-squares on $F^{2}$ \\
Hydrogens & Difference map \\
Data/restraints/parameters & $3557 / 0 / 325$ \\
Goodness-of-fit on $F^{2}$ & 1.089 \\
Final $R$ index $[I>2 \sigma(I)]$ & $R_{1}=0.0410$ \\
Final $R$ indices $($ all data $)$ & $R=0.0488, w R_{2}=0.1371$ \\
Largest diff. peak and hole & $0.130 /-0.170$ \\
\hline \hline
\end{tabular}

crystallographic parameters and summary of structure refinement are tabulated in Tables II and III, respectively. Crystallographic data have been deposited with Cambridge Crystallographic Data Centre: Deposition No. CCDC-957121. Copies of the data can be obtained free of charge via http://www.ccdc.cam.ac.uk/conts/retrieving.html (or from the Cambridge Crystallographic Data Centre, 12, Union Road, Cambridge CB2 1EZ, UK; Fax: +44 1223 336033; e-mail: deposit@ccdc.cam.ac.uk.

The final structure reached is depicted in Fig. 6. No appreciable disorder was found in the nonyl chain, in accordance with the thermodynamic analysis. ${ }^{7,8}$ The structure consists of layers formed by dimers related by the inversion. The figure axis of dimers is oblique to the layer. As for the internal structure of a dimer, the so-called $\pi-\pi$ interaction between biphenyl cores seems active, in contrast to the strong interaction between electric dipoles of cyano (NC-) groups in ordered crystals of $n \mathrm{CBs},{ }^{56-64}$ which has a cyano group at one end of the biphenyl core in place of the $\mathrm{SCN}-$ group in $n \mathrm{TCB}$.

${ }^{1}$ Handbook of Liquid Crystals, edited by D. Demus, J. Goodby, G. W. Gray, H. W. Spiess, and V. Vill (Wiley-VCH, Weinheim, Germany, 1998), Vol. 1. ${ }^{2}$ The Plastically Crystalline State: Orientationally-Disordered Crystals, edited by J. N. Sherwood (John Wiley \& Sons Ltd., Chichester, UK, 1979).

${ }^{3}$ S. Ishimaru, K. Saito, S. Ikeuchi, M. Massalska-Arodź, and W. Witko, J. Phys. Chem. B 109, 10020 (2005).

${ }^{4}$ M. Funahashi and J. Hanna, Mol. Cryst. Liq. Cryst. 368, 303 (2001).

${ }^{5}$ W. Pisula, M. Zorn, J.-Y. Chang, K. Mullen, and R. Zentel, Macromol. Rapid. Commun. 30, 1179 (2009).

${ }^{6}$ M. Funahashi and J. Hanna, Appl. Phys. Lett. 73, 3733 (1998).

${ }^{7}$ K. Horiuchi, Y. Yamamura, R. Pełka, M. Sumita, S. Yasuzuka, M. Massalska-Arodź, and K. Saito, J. Phys. Chem. B 114, 4870 (2010).

${ }^{8}$ Y. Yamamura, T. Adachi, T. Miyazawa, K. Horiuchi, M. Sumita, M. Massalska-Arodź, S. Urban, and K. Saito, J. Phys. Chem. B 116, 9255 (2012).

${ }^{9}$ T. Adachi, Y. Yamamura, M. Hishida, M. Ueda, S. Ito, and K. Saito, Liq. Cryst. 39, 1340 (2012).

${ }^{10}$ T. Adachi, H. Saitoh, Y. Yamamura, M. Hishida, M. Ueda, S. Ito, and K. Saito, Bull. Chem. Soc. Jpn. 86, 1022 (2013).

${ }^{11}$ Y. Yamaoka, Y. Taniguchi, S. Yasuzuka, Y. Yamamura, and K. Saito, J. Chem. Phys. 135, 044705 (2011).
${ }^{12}$ Y. Nakazawa, Y. Yamamura, S. Kutsumizu, and K. Saito, J. Phys. Soc. Jpn. 81, 094601 (2012).

${ }^{13}$ K. Saito and M. Sorai, Chem. Phys. Lett. 366, 56 (2002).

${ }^{14}$ K. Saito, Pure Appl. Chem. 81, 1783 (2009).

${ }^{15}$ M. Massalska-Arodź, A. Würflinger, and D. Büsing, Z. Naturforsch. 54a, 675 (1999).

${ }^{16}$ S. Urban, K. Czuprynski, R. Dabrowski, B. Gestblom, J. Janik, H. Kresse, and H. Schmalfuss, Liq. Cryst. 28, 691 (2001).

${ }^{17}$ M. Massalska-Arodź, H. Schmalfuss, W. Witko, H. Kresse, and A. Würflinger, Mol. Cryst. Liq. Cryst. 366, 221 (2001).

${ }^{18}$ A. Würflinger and S. Urban, Liq. Cryst. 29, 799 (2002).

${ }^{19}$ S. Urban, J. Czub, R. Dabrowski, and H. Kresse, Liq. Cryst. 32, 119 (2005).

${ }^{20}$ S. Urban, J. Czub, R. Dabrowski, and A. Würflinger, Phase Transitions 79, 331 (2006).

${ }^{21}$ M. Jasiurkowska, A. Budziak, J. Czub, and S. Urban, Acta Phys. Pol. A 110, 795 (2006).

${ }^{22}$ R. Pełka, Y. Yamamura, M. Jasiurkowska, M. Massalska-Arodź, and K. Saito, Liq. Cryst. 35, 179 (2008).

${ }^{23}$ M. Jasiurkowska, A. Budziak, J. Czub, M. Massalska-Arodź, and S. Urban, Liq. Cryst. 35, 513 (2008).

${ }^{24}$ C. M. Roland, R. B. Bogoslovov, R. Casalini, A. R. Ellis, S. Bair, S. J. Rzoska, K. Czuprynski, and S. Urban, J. Chem. Phys. 128, 224506 (2008).

${ }^{25}$ M. Jasiurkowska, J. Ściesiński, J. Czub, M. Massalska-Arodź, R. Pełka, E. Juszyńska, Y. Yamamura, and K. Saito, J. Phys. Chem. B 113, 7435 (2009).

${ }^{26}$ Y. Maeda and S. Urban, Phase Transitions 83, 467 (2010).

${ }^{27}$ M. Jasiurkowska, P. Zieliński, M. Massalska-Arodź, Y. Yamamura, and K. Saito, J. Phys. Chem. B 115, 12327 (2011).

${ }^{28}$ S. Urban and C. M. Roland, J. Non-Cryst. Solids 357, 740 (2011).

${ }^{29}$ T. Miyazawa, Y. Yamamura, M. Hishida, S. Nagatomo, M. MassalskaArodź, and K. Saito, J. Phys. Chem. B 117, 8293 (2013).

${ }^{30}$ S. Diele, S. Tosch, S. Mahnke, and D. Demus, Cryst. Res. Technol. 26, 809 (1991).

${ }^{31}$ T. Ishiguro, K. Yamaji, and G. Saito, Organic Superconductors (SpringerVerlag, Heidelberg, Germany, 1998).

${ }^{32}$ S. Pensec, F.-G. Tournilhac, and P. Bassoul, J. Phys. II 6, 1597 (1996).

${ }^{33}$ M. Sorai and K. Saito, Chem. Rec. 3, 29 (2003).

${ }^{34}$ A. Sato, Y. Yamamura, K. Saito, and M. Sorai, Liq. Cryst. 26, 1185 (1999).

${ }^{35}$ K. Saito, T. Shinhara, T. Nakamoto, S. Kutsumizu, S. Yano, and M. Sorai, Phys. Rev. E 65, 031719 (2002).

${ }^{36}$ K. Saito, M. Ikeda, and M. Sorai, J. Therm. Anal. 70, 345 (2002).

${ }^{37}$ S. Kutsumizu, H. Mori, M. Fukatami, and K. Saito, J. Appl. Crystallogr. 40, s279 (2007).

${ }^{38}$ S. Kutsumizu, H. Mori, M. Fukatami, S. Naito, K. Sakajiri, and K. Saito, Chem. Mater. 20, 3675 (2008).

${ }^{39}$ F. Dowell, Phys. Rev. A 28, 3526 (1983).

${ }^{40}$ See supplementary material at http://dx.doi.org/10.1063/1.4821162 for the calculation of the form factor of box-shaped electron density with rounding and discussions on its properties and implications.

${ }^{41}$ M. J. Frisch, G. W. Trucks, H. B. Schlegel et al., Gaussian 09, Revision A.1, Gaussian, Inc., Wallingford, CT, 2009.

${ }^{42}$ K. Ozawa, Y. Yamamura, S. Yasuzuka, H. Mori, S. Kutsumizu, and K. Saito, J. Phys. Chem. B 112, 12179 (2008).

${ }^{43}$ K. Saito, M. Okada, H. Akutsu, and M. Sorai, Chem. Phys. Lett. 318, 75 (2000).

${ }^{44}$ K. Saito, M. Okada, H. Akutsu, A. Sato, and M. Sorai, J. Phys. Chem. B 108, 1314 (2004).

${ }^{45}$ A. J. Leadbetter, J. L. A. Durand, and M. Rugman, Mol. Cryst. Liq. Cryst. 34, 231 (1976).

${ }^{46}$ A. J. Leadbetter, J. C. Frost, J. P. Gaughan, G. W. Gray, and A. Mosley, J. Phys. (Paris) 40, 375 (1979).

${ }^{47}$ S. Asakura and F. Oosawa, J. Chem. Phys. 22, 1255 (1955).

${ }^{48}$ J. Alder and T. E. Wainwright, J. Chem. Phys. 27, 1208 (1957).

${ }^{49}$ L. Onsager, Ann. N. Y. Acad. Sci. 51, 627 (1949).

${ }^{50}$ P. Bolhuis and D. Frenkel, J. Chem. Phys. 106, 666 (1997).

${ }^{51}$ J. A. C. Veerman and D. Frenkel, Phys. Rev. A 43, 4334 (1991).

${ }^{52}$ G. J. Krüger, Phys. Rep. 82, 229 (1982).

${ }^{53}$ PROCESS-AUTO, Rigaku Corporation, Tokyo, Japan, 1998.

${ }^{54}$ M. C. Burla, M. Camalli, G. Cascarano, C. Giacovazzo, G. Polidori, R. Spagna, and D. Viterbo, J. Appl. Crystallogr. 22, 389 (1989).

${ }^{55}$ G. M. Sheldrick, Acta Crystallogr., Sect. A: Found. Crystallogr. A64, 112 (2008).

${ }^{56}$ W. Haase, J. Loub, and H. Paulus, Z. Krystallogr. 202, 7 (1992).

${ }^{57}$ W. Haase, H. Paulus, and R. Redzialer, Mol. Cryst. Liq. Cryst. 100, 211 (1983). 
${ }^{58}$ G. V. Vani, Mol. Cryst. Liq. Cryst. 99, 21 (1983).

${ }^{59}$ T. Hanemann, W. Haase, I. Svodoba, and H. Fuess, Liq. Cryst. 19, 699 (1995).

${ }^{60}$ M. Kuribayashi and K. Hori, Liq. Cryst. 26, 809 (1999).

${ }^{61}$ M. Kuribayashi and K. Hori, Acta Crystallogr., Sect. C: Cryst. Struct. Commun. 54, 1475 (1998)
${ }^{62}$ T. Manisekaran, R. K. Bamezai, N. K. Sharma, and J. S. Prasad, Liq. Cryst. 23, 597 (1997)

${ }^{63}$ T. Manisekaran, R. K. Bamezai, N. K. Sharma, and J. S. Prasad, Mol. Cryst. Liq. Cryst. 268, 45 (1995).

${ }^{64}$ T. Manisekaran, R. K. Bamezai, N. K. Sharma, and J. S. Prasad, Mol. Cryst. Liq. Cryst. 268, 83 (1995). 